\title{
Philosophiques
}

\section{Sur la portée des prérogatives personnelles}

\section{Idil Boran}

Volume 32, numéro 2, automne 2005

URI : https://id.erudit.org/iderudit/011871ar

DOI : https://doi.org/10.7202/011871ar

Aller au sommaire du numéro

Éditeur(s)

Société de philosophie du Québec

ISSN

0316-2923 (imprimé)

1492-1391 (numérique)

Découvrir la revue

Citer cet article

Boran, I. (2005). Sur la portée des prérogatives personnelles. Philosophiques, 32(2), 343-355. https://doi.org/10.7202/011871ar d'utilisation que vous pouvez consulter en ligne.

https://apropos.erudit.org/fr/usagers/politique-dutilisation/ 


\title{
Sur la portée des prérogatives personnelles
}

\author{
IDIL BORAN \\ Département de philosophie \\ Université York et Uqam \\ boran.idil@uqam.ca
}

Les prérogatives personnelles sont des libertés accordées aux individus afin qu'ils puissent poursuivre leurs propres intérêts, projets, et ambitions. La question morale que cela soulève est de savoir jusqu'à quel point ces permissions personnelles peuvent aller. Quelle est la portée des prérogatives personnelles et quelles en sont les limites? Dans ses travaux, Samuel Scheffler soutient l'idée que la justification des prérogatives personnelles est fondée sur l'indépendance du point de vue personnel et que cette justification n'est pas soumise aux restrictions déontologiques. C'est cette thèse que l'auteur a appelée la thèse d'indépendance. Il en découle qu'il est possible d'accepter des prérogatives sans restrictions, ce qui est la thèse d'asymétrie. Même si la thèse d'indépendance a suscité des réactions critiques, celles-ci se limitent aux arguments négatifs qui mettent sa validité en cause. Mon objectif, dans cet article, est de poursuivre ce débat et d'offrir des arguments positifs qui montreront que la justification des prérogatives personnelles dépend en fait des restrictions déontologiques. Mon analyse montrera, contre Scheffler, que toute justification des prérogatives personnelles implique une justification des restrictions déontologiques.

Mon analyse sera structurée de la façon suivante. Je commencerai par une mise en contexte de la problématique où il s'agira d'expliquer que la justification des prérogatives personnelles se situe dans un projet de rejet du conséquentialisme. La deuxième section étalera la thèse d'indépendance soutenue par Scheffler qui sera suivie, dans la troisième section, par un exposé de la thèse d'asymétrie. La quatrième section sera consacrée aux objections qui, par des arguments négatifs, mettent en cause la thèse d'indépendance. Dans la section suivante, j'offrirai deux arguments positifs en faveur de ce que je nomme "une thèse de dépendance». Le premier argument en appelle aux droits fondamentaux. Il vise à démontrer que la protection des prérogatives requiert un système de restrictions déontologiques. Le second est un argument contractualiste qui montre que les prérogatives peuvent engendrer le dilemme du prisonnier dans des situations à plusieurs acteurs. Tous les deux convergeront vers la conclusion que toute justification de prérogatives personnelles dépend, pour qu'elle puisse tenir, des restrictions déontologiques. La sixième section anticipera une objection contre ma thèse et offrira une réponse.

\section{Le conséquentialisme et ses critiques}

Les prérogatives personnelles ont une place importante dans les théories morales modernes. Elles sont souvent invoquées par opposition aux théories 
conséquentialistes. Le conséquentialisme requiert de l'agent moral de toujours choisir l'action qui contribuera le plus au bien général conçu d'un point de vue impartial. L'importance qu'elle attribue au point de vue impartial constitue l'attrait de cette théorie. Historiquement, les positions morales conséquentialistes (dont l'utilitarisme) ont été reconnues comme étant progressistes, égalitaires, et modernes.

Cependant, l'exigence d'impartialité entraîne une exigence particulière propre aux théories conséquentialistes. La maximisation du bien général conçu d'un point de vue impartial implique que l'on attend des agents moraux de sacrifier leurs propres intérêts ou projets afin d'y contribuer. Même si l'importance qu'il donne au point de vue impartial est souvent louée, le conséquentialisme a suscité un certain nombre d'objections qui mettent en doute sa plausibilité en tant que théorie morale.

Deux objections sont souvent soulevées. L'une consiste à dire que le conséquentialisme est trop exigeant, car il requiert des agents de sacrifier leurs propres intérêts ou projets afin de contribuer au bien général. L'autre consiste à dire que le conséquentialisme n'est pas suffisamment exigeant, car certaines actions inacceptables selon certains de nos jugements considérés justes seraient permises par le conséquentialiste s'il se trouvait qu'elles contribuent au bien général. Le conséquentialisme permettrait par exemple de nier les intérêts fondamentaux de certains pour apporter un plus grand bien à un plus grand nombre de personnes. Ces objections ont incité beaucoup de philosophes à rejeter le conséquentialisme ou du moins à le critiquer sévèrement. Mais le rejet de cette théorie n'est pas monolithique. Ce que montrent les écrits de certains philosophes (p. ex. Scheffler, Nagel, Heyd, Kagan), c'est qu'il ne suffit pas de la rejeter et de la remplacer par une forme quelconque du non-conséquentialisme, mais de clarifier quelle forme du non-conséquentialisme serait la plus plausible.

Une approche particulière qui consiste à prendre une distance du conséquentialisme est celle qui tente de motiver les prérogatives personnelles. Par prérogatives personnelles, on entend les libertés qui permettent à l'agent d'agir quelquefois de manière non optimale d'un point de vue impartial pour se tourner vers ses propres projets et intérêts, désirs et ambitions. Les prérogatives personnelles ne sont pas les seuls résultats d'un raisonnement non conséquentialiste. À la lumière de la deuxième objection, il est possible de formuler des restrictions déontologiques, dont le rejet ne serait pas permis même dans des circonstances où il apporterait un résultat optimal d'un point de vue impartial. La façon dont les prérogatives personnelles et les restrictions déontologiques sont intégrées à une conception morale donne à cette conception son positionnement spécifique à l'intérieur du cadre non conséquentialiste.

Dans son ouvrage, The Rejection of Consequentialism, Samuel Scheffler défend une version du non-conséquentialisme qui justifie les prérogatives personnelles. La particularité de la position de l'auteur, c'est de justifier les 
prérogatives sans justification correspondante des restrictions déontologiques. Scheffler appelle cette position «la thèse d'asymétrie».

\section{Scheffler et l'indépendance des prérogatives personnelles}

Dans The Rejection of Consequentialism, Scheffler recherche une justification des prérogatives personnelles. Il reconnaît qu'il y a deux stratégies permettant de mettre en cause le conséquentialisme ; l'une est la justification des prérogatives personnelles et l'autre est la justification des restrictions déontologiques. Pour Scheffler, une conception dite « hybride », fondée sur la justification des prérogatives personnelles, est possible, par contraste à une conception "complète" qui inclurait les deux stratégies.

L'objectif de Scheffler est d'abord de fournir une justification plausible des prérogatives personnelles. Une prérogative personnelle serait la liberté accordée à l'agent de poursuivre ses propres intérêts et projets sans égard à la poursuite d'un résultat optimal selon un calcul impartial. La thèse principale d'un non-conséquentialisme qui se fonderait sur ces prérogatives serait de dire qu'il est parfois admissible de ne pas agir de façon à promouvoir un résultat optimal d'un point de vue impartial. Pour illustrer cette thèse, imaginons une personne, Sophie. Si Sophie était conséquentialiste, elle donnerait pour des raisons morales une grande partie de ses biens matériels, par exemple, afin de combattre la pauvreté, quels que soient ses propres plans ou engagements. Elle consacrerait aussi une grande partie de son temps à travailler, sans doute, pour une organisation contre la pauvreté. Imaginez maintenant que Sophie soit étudiante en musique et aspire à devenir violoncelliste. Le non-conséquentialisme fondé sur les prérogatives personnelles affirme qu'il est parfois admissible de ne pas agir de façon à promouvoir un résultat optimal d'un point de vue impartial. Dans ce cadre, il serait alors permis à Sophie de se consacrer à ses propres projets (par ex. celui de devenir une violoncelliste virtuose). Fondée sur les prérogatives personnelles, une telle conception hybride du non-conséquentialisme permettrait à l'agent de promouvoir le bien s'il choisissait d'agir ainsi. Si Sophie le choisit, elle peut toujours consacrer son temps à l'organisation contre la pauvreté. Mais un tel acte ne serait pas, contrairement à ce que le conséquentialisme prescrit, une exigence morale.

Afin de donner une justification rationnelle des prérogatives personnelles, Scheffler se concentre sur l'indépendance du point de vue personnel. La présupposition méta-théorique (ou méta-éthique) derrière cette manœuvre est qu'une conception éthique présuppose toujours une représentation - au moins minimale - de ce qu'est une personne. Le raisonnement, tel qu'articulé par Scheffler, se développe ainsi:

i. Chaque individu a un point de vue personnel de sorte que ses projets, ambitions et intérêts ont un statut spécial; il veille sur ses projets bien plus qu'au bien général. 
ii. Le point de vue personnel est indépendant du point de vue impersonnel.

iii. Si une théorie morale se veut plausible, elle doit tenir compte de l'indépendance des personnes.

iv. Une façon de tenir compte de l'indépendance des personnes serait d'adopter une stratégie de «libéralisation individuelle», ce qui donnerait lieu à des prérogatives personnelles permettant à l'agent de poursuivre ses propres projets de façon disproportionnelle à un calcul impersonnel.

v. Conclusion: il existe une raison plausible de reconnaitre les prérogatives personnelles.

L'inclusion des prérogatives dans une théorie éthique représente, selon ce raisonnement, une stratégie rationnelle pour prendre en considération l'indépendance du point de vue personnel. Par contre, il faut noter tout de suite que la stratégie de la libéralisation individuelle n'est pas la seule stratégie qui reconnaisse le point de vue personnel. Scheffler convient qu'il existe d'autres stratégies, et certaines d'entre elles sont utilisables par le conséquentialiste. Notre raisonnement tente de montrer que si cette stratégie est également adéquate, elle nous donne une justification plausible de la reconnaître.

La reconnaissance du point de vue personnel donne lieu à ce que Scheffler appelle la "thèse d'indépendance» (the independence thesis). Selon cette thèse la justification des prérogatives personnelles serait indépendante de toute perspective impartiale. Dans notre exemple hypothétique, l'ambition professionnelle de Sophie pourrait donc se justifier en invoquant l'indépendance de son point de vue personnel par rapport à toute optique impartiale. Consacrer son temps, ses efforts, et des biens matériels à la poursuite de ses ambitions de violoncelliste ferait partie des prérogatives personnelles de Sophie, étant donné l'indépendance du point de vue personnel.

\section{La thèse d'asymétrie}

D'emblée, la question suivante se pose: pourquoi une justification des prérogatives n'impliquerait-elle pas l'approbation de certaines restrictions déontologiques? Puisque les prérogatives personnelles d'un côté et les restrictions déontologiques de l'autre représentent deux façons de rejeter le conséquentialisme, il semble évident de penser que les deux éléments pourraient donner une conception complète du non-conséquentialisme. Or les conclusions de Scheffler vont à l'encontre de cette supposition. Après avoir justifié la plausibilité des prérogatives en faisant appel à la thèse d'indépendance, Scheffler poursuit le même questionnement pour les restrictions déontologiques et affirme qu'il n'existe pas de justification correspondante pour celles-ci. Ainsi, Scheffler soutient la thèse selon laquelle un agent peut accepter une prérogative et refuser en même temps les restrictions déontologiques. 
L'intuition qui sous-tend la thèse d'asymétrie est la suivante. La justification des prérogatives est indépendante de toute considération d'un point de vue impartial. Or, contrairement aux prérogatives, les restrictions déontologiques font appel à un point de vue impartial. Si la justification des prérogatives est indépendante de tout point de vue impartial, elle devrait aussi être indépendante de la justification des restrictions déontologiques. Cela veut dire qu'une telle justification ne peut pas faire partie d'un raisonnement déontologique. En effet, Scheffler insiste sur ce point dans sa défense des prérogatives: "The underlying principled motivation for an agent-centred prerogative is independent of any rationale there may be for agent-centred restrictions» (p. 81). Le résultat est la thèse d'asymétrie: un agent moral peut accepter des prérogatives personnelles tout en refusant les restrictions déontologiques.

\section{Objections à la thèse d'asymétrie}

La thèse d'asymétrie a suscité maintes réactions critiques. Nous pouvons identifier deux reproches principaux. Le premier affirme que la thèse d'asymétrie permettrait des actions inacceptables selon nos jugements considérés comme justes. Ce reproche a été soulevé par Shelley Kagan ${ }^{1}$. Dans cette optique, même si l'on reconnaît l'attrait intuitif de l'indépendance du point de vue personnel, au moment où l'on arrive à la thèse d'asymétrie, on se heurte à des propositions très problématiques. La thèse d'asymétrie donnerait à l'agent la permission de commettre des actes qui causeraient du tort à d'autres si ces actes-là faisaient partie de ses engagements et de ses projets. Kagan écrit: "such a prerogative will not only permit agents to allow harm, it will also permit agents to do harm in the pursuit of their non-optimal projects $^{2} »$. Ce que Kagan reproche à la thèse d'asymétrie, c'est qu'il serait permis, dans les circonstances appropriées, de tuer une personne pour pouvoir hériter de $10000 \$$. Cela est un résultat pervers qui va à l'encontre de nos intuitions morales les plus fondamentales. Par exemple, serait-il justifié que, la veille de l'audition pour l'admission à la symphonie, Sophie mette du poison dans la boisson de son compétiteur, ce qui le rendra malade pour quelques jours? Cette action satisfait toutes les conditions du problème que soulève Kagan. Elle permet à Sophie de maximiser ses chances quant à ses ambitions personnelles, elle est non optimale au sens où elle n'apporte pas un plus grand bien pour tout le monde (en d'autres mots cette action n'a d'utilité que pour Sophie, si on suppose que le talent des deux compétiteurs est comparable). Et elle va à l'encontre de nos intuitions morales.

1. D'autres objections ont été faites par Stephen Darwall, dans sa recension de The Rejection of Consequentialism, The Journal of Philosophy, 81, 1984, p. 220-226. Pour une plus récente objection, voir Ramon Das, "Prerogatives without Restrictions? ", Philosophical Studies, 99, 2000, p. 347-372.

2. Kagan, 1984, p. 249. 
Il est possible, à ce stade-ci, de venir à la défense de Scheffler en affirmant que les prérogatives sont permises à l'intérieur de certaines limites. Il ne s'agit pas de prérogatives illimitées. Scheffler offre un critère qui définit les limites des prérogatives. Une prérogative permet à l'agent de se concentrer sur ses propres projets et engagements, à moins que le degré de ce qui est non optimal d'un point de vue impartial dépasse un degré limite défini par un point de vue impartial et accepté par le conséquentialiste ${ }^{3}$. Mais le problème qui a été identifié demeure, car, à l'intérieur de ces limites, les prérogatives permettront quand même des actes de malfaisance. Certes, si le compétiteur de Sophie est malade pendant quelques jours, cela ne constituera pas une grande perte pour la communauté artistique (si l'on suppose que tous les deux ont un talent comparable). Mais l'action semble quand même injuste indépendamment des conséquences générales. Le problème soulevé ici n’est pas que les prérogatives sont illimitées, mais plutôt que les limites des prérogatives ne sont pas définies par des contraintes déontologiques. Le moyen d'empêcher ce résultat serait, bien sûr, d'imposer des restrictions déontologiques, mais la thèse d'asymétrie ne permet pas les restrictions déontologiques.

Une deuxième objection vient de Stephen Darwall. L'objection de celuici ne consiste pas à analyser directement les limites des prérogatives, mais plutôt à questionner leur indépendance. Dans cette optique, les limites des prérogatives s'imposent de façon dérivative, par l'intermédiaire du questionnement de la thèse d'indépendance ${ }^{4}$. Selon Darwall, si les prérogatives étaient vraiment indépendantes, la théorie morale permettrait alors le sacrifice de toute valeur impersonnelle, puisqu'une indépendance absolue empêcherait toute comparaison entre les raisons qui justifient les prérogatives personnelles et toutes raisons impersonnelles, que ce soit les raisons prescrites par le conséquentialisme ou celles des restrictions déontologiques. Ainsi, Darwall écrit : "What is needed is a justificatory standpoint from which one can balance the competing rationales, but it is far from clear what that would be $e^{5}$. Le problème soulevé est donc un problème d'incommensurabilité qui surgit de la thèse d'indépendance.

En somme, Kagan et Darwall offrent des arguments différents, mais ils aboutissent, comme le remarque aussi Das (2000), à des conclusions semblables. Tous les deux sont préoccupés par la portée (élargie) des prérogatives par appel à la thèse d'indépendance. Ces objections se limitent à offrir des arguments négatifs dont le but est de montrer l'insuffisance de la thèse d'asymétrie. Je tenterai de reprendre là où ces critiques s'arrêtent et de développer une thèse positive dont le but sera de fournir une justification des prérogatives personnelles qui éviterait les problèmes soulevés. Cette thèse, que j'appellerai «thèse de dépendance », vise à montrer qu'une justification

3. Scheffler, 1982, p. 20; Kagan, 1984, 250-251; Das, 2000.

4. Darwall, 1984; Das, 2000, 360.

5. Darwall, 1984, 224. 
plausible des prérogatives doit faire appel à des restrictions déontologiques. Il en résulte, par contre, que les prérogatives seront, dans cette optique, plus limitées que ce qui découle de la thèse d'asymétrie défendue par Scheffler.

\section{Deux arguments en faveur de la thèse de dépendance}

Deux arguments en faveur de la dépendance des prérogatives personnelles aux restrictions déontologiques sont disponibles. Je les appellerai respectivement «l'argument par appel aux droits fondamentaux » et «l'argument contractualiste».

\section{L'argument par appel aux droits fondamentaux}

Le premier argument justifie la thèse de la dépendance des prérogatives aux restrictions déontologiques en faisant appel aux droits fondamentaux. S'il est juste, cet argument montrera que la justification des prérogatives personnelles ne peut se faire sans référence déontologique à la protection des droits fondamentaux. En tant qu'individus nous avons des projets et des engagements qui sont les nôtres et forment notre «point de vue personnel ». C'est aussi ce que Scheffler affirme dans (i). Les projets et les engagements reflètent les intérêts des personnes. Or il existe une corrélation entre les intérêts et les droits. Les intérêts peuvent fonder des droits, qui restreindraient partiellement le champ d'action d'autrui. De là, on peut aboutir à une justification de certaines prérogatives personnelles.

Avoir un point de vue personnel, c'est avoir des intérêts. Mais si ces intérêts peuvent fonder des droits, cela veut dire que certaines restrictions déontologiques devraient être respectées par tout le monde. Concevoir les prérogatives de cette façon implique donc que le raisonnement ait une dimension déontologique. Si ce raisonnement est valide, il montre qu'en partant de la prémisse principale de Scheffler, qui affirme le point de vue personnel, on aboutit à des restrictions déontologiques.

Dans cette optique, garantir les prérogatives, c'est accepter des restrictions déontologiques. Cela donne lieu à une proposition P1:

P1: Toute justification d'une prérogative dont disposerait un agent moral comprend un élément déontologique.

Cette proposition ne nie pas, mais reconnaît plutôt la singularité du point de vue personnel. Ce n'est pas la singularité du point de vue personnel en soi qui justifie les prérogatives. L'agent a droit à des prérogatives parce que celles-ci protègent les intérêts les plus fondamentaux des personnes. Selon ce raisonnement, ne pas reconnaître le point de vue personnel, c'est nier les intérêts des personnes. Si les intérêts sont corrélatifs aux droits, les individus peuvent revendiquer leur droit à l'espace personnel.

Cette conception des droits moraux où les droits sont corrélatifs aux intérêts est défendue par certains philosophes, notamment Joel Feinberg (1980) et Joseph Raz (1984). Pour Feinberg, par exemple, avoir un droit, 
c'est avoir des intérêts ${ }^{6}$. Les intérêts fondent les droits, et les droits justifient des obligations envers d'autres. Ces droits sont des droits, non pas envers des personnes spécifiques, mais envers tout le monde, juste par le fait que chaque personne a un ensemble d'intérêts qui lui sont propres? ${ }^{7}$. Feinberg les appelle les droits in $\mathrm{rem}^{8}$. Pour ce type de droits, une obligation est imposée à toute personne de respecter l'espace personnel de l'agent. Donc, notre agent moral hypothétique, Sophie, peut revendiquer ses droits à diriger sa vie de l'intérieur, c'est-à-dire de façon autonome. Cela imposerait à toute autre personne de respecter l'espace personnel dans lequel elle pourrait exercer son autonomie en tant qu'agent.

Cette façon de justifier les prérogatives est différente de celle de Scheffler sur un point important. Rappelons que l'ambition de Scheffler est modeste. Il ne tente pas de démontrer que les prérogatives devraient nécessairement être reconnues, il se contente de démontrer qu'il y a une raison plausible de les reconnaître. Or la proposition P1 ne se contente pas de permettre les prérogatives, elle garantit un certain nombre de prérogatives.

Si cette proposition est juste, une proposition P2 s'en suit:

P2: Toute prérogative acceptée par un agent moral impose une restriction à cet agent.

Si toute personne a droit à un espace personnel (garanti par la première proposition), cela impose des restrictions à toute autre personne. Par conséquent, le droit des autres personnes à leur propre espace moral imposerait simultanément des restrictions à l'agent. Si cette proposition est juste, toute acceptation d'une prérogative impose à l'agent des restrictions déontologiques. Ainsi, la justification des prérogatives n'engendre pas la permission de choisir n'importe quelle action à l'intérieur des limites du conséquentialisme afin de réaliser des projets individuels.

Il découle de ce raisonnement que l'acceptation de prérogatives ne peut donc pas se faire indépendamment des restrictions déontologiques. Accepter les prérogatives personnelles, c'est accepter en même temps des restrictions déontologiques qui restreignent le champ des prérogatives. Il n'y aurait pas moyen de garantir les prérogatives s'il n'y avait pas de restrictions déontologiques pour les protéger. C'est ainsi même si on accepte la singularité du point de vue personnel. Si j'ai raison, un agent ne peut pas, contrairement à ce que Scheffler affirme, justifier les prérogatives personnelles tout en rejetant les restrictions déontologiques.

6. Feinberg, 1980, p. 159-184.

7. Feinberg, 1980, p. 134.

8. Ces droits s'opposent aux droits qui s'appliqueraient aux personnes spécifiques dans des situations contingentes, comme celles qui proviennent d'une entente. 


\section{L'argument contractualiste}

Le deuxième argument en faveur des prérogatives personnelles qui dépendent des restrictions déontologiques est ce que j'appellerai «l'argument contractualiste ». La justification des prérogatives personnelles fournie par Scheffler a une structure particulière. Elle suppose que les agents agissent en fonction d'actions isolées et que le choix ne se fasse qu'en tenant compte des désirs de l'individu et de la situation dans laquelle il se trouve. Dans la terminologie contractualiste, cette situation de choix est appelée "paramétrique " ${ }^{9}$. Selon cette optique, l'agent est le seul centre de l'action ${ }^{10}$. Certes, les décisions paramétriques représentent certaines de nos décisions. Par exemple, si Agathe a le choix entre travailler sur son manuscrit ou partir en vacances, elle peut prendre sa décision strictement en fonction de ses préférences. Ce serait une décision paramétrique.

Cependant, dans la plupart des contextes sociaux, les décisions des individus se prennent par rapport aux actions des autres. Dans ce cas, il est impossible de prendre une décision sur une action sans tenir compte des attentes que l'on pourrait avoir en ce qui concerne les actions des autres acteurs. C'est l'approche de la théorie des jeux qui est au centre de certaines formes de l'éthique contractualiste, notamment le contractualisme de Gauthier. Par exemple, Aude doit décider si elle ira à la fête d'Aurélie à laquelle elle est invitée. Si Tom est là, elle préfère ne pas y aller. Mais si Jonathan y est, elle ira certainement, même si Tom est là. La décision qu'Aude prendra dépend en partie des décisions que prennent Aurélie (quand elle fait sa liste d'invités), Jonathan, et Tom. Pour prendre une décision, Aude aura donc besoin de l'information sur les décisions que prennent ces personnes, ce qui lui permettra de faire un calcul de probabilité et d'en faire une évaluation rationnelle à la lumière de ses propres préférences.

Quand ce facteur est pris en considération, la question de la justification des prérogatives personnelles prend une nouvelle dimension. Les prérogatives ne peuvent pas toujours être justifiées en tant que telles, c'est-à-dire en considérant seulement leur attrait pour l'agent dans une situation paramétrique. Beaucoup de situations font que les prérogatives ne peuvent être considérées qu'en tenant compte de ce que l'on peut attendre des autres agents. Elles devraient être conçues sur une plateforme interactive. Un des désavantages du raisonnement de Scheffler, c'est qu'il est uniquement paramétrique et ne tient pas compte des situations interactives, qui constituent une grande partie des situations d'action.

Or, dans les situations interactives, une version du dilemme du prisonnier surgit de l'application des prérogatives personnelles sans restrictions. En tant qu'agents, nous nous trouvons souvent dans des situations où les actions de certains imposent des coûts aux autres. Cela s'applique aussi aux 
prérogatives. Si un individu agit selon ses prérogatives personnelles, il se peut que ses actions imposent des coûts aux autres. Dans ces cas, les autres personnes se mettront dans une position concurrentielle et agiront de leur côté de façon à éviter ces coûts ou, si ce n'est pas possible, en imposant autant de coûts en réponse pour égaliser les coûts et les bénéfices. Mais, dans ces cas-là, la distribution des coûts et des bénéfices n'est certainement pas "égalitaire ». Elle n'est pas fondée sur un principe moral quelconque. Elle est plutôt de nature concurrentielle.

Par exemple, imaginons que je brûle du bois dans mon jardin et que je justifie cette action en faisant appel à mes prérogatives personnelles, qui me permettent d'agir de façon disproportionnelle au bien général conçu d'un point de vue impartial. Mon action est une nuisance pour mes voisins: je pollue l'air, propulse des cendres dans leur jardin. Cela réduit les bénéfices que mes voisins peuvent tirer de leur propre jardin (passer des soirées sur des chaises longues en famille ou entre amis, passer les fins de semaine à s'occuper paisiblement du jardin, etc.). S'il ne peut pas me convaincre de ne plus brûler de bois, le voisin d'à côté peut bien se mettre à pratiquer son hobby préféré qui est de faire de la menuiserie en plein air en utilisant des outils bruyants ou organiser des fêtes retentissantes jusqu'au petit matin. Rien ne l'empêcherait de raisonner de cette façon. Si, de mon côté, je salis son jardin par mes activités et n'offre rien en compensation, il n'y a aucune raison qu'il s'empêche de poursuivre ses activités bruyantes.

Toutefois, par ce raisonnement, le caractère purement concurrentiel de la distribution de coûts et des bénéfices est non seulement moralement suspect, mais aussi irrationnel. Il serait à l'avantage des deux voisins que chacun évite (ou minimise) les coûts qu'il impose à l'autre par ses activités. Mais aucun des deux voisins n'est motivé à imposer des restrictions à ses propres actions à cause de la nature concurrentielle de leur interaction. Chacun des voisins pense que s'il restreint ses activités il en sortira perdant. Donc, tous les deux continuent à revendiquer leurs prérogatives sans restrictions et créent une situation nocive pour l'autre. Dans ce cas-là, les deux voisins sont moyennement satisfaits par le fait qu'aucun des deux n'est perdant, mais leur satisfaction est réduite à cause des externalités nocives qu'ils s'infligent mutuellement. Et s'ils continuent à augmenter le degré d'externalité qu'ils produisent, il arrivera un moment où ils ne pourront même plus exercer leurs propres prérogatives. Le bruit risque de devenir insupportable au point où il empêchera le voisin de passer son temps dans le jardin à brûler du bois, et en revanche les cendres et la fumée peuvent rendre la menuiserie en plein air impossible à pratiquer. Par conséquent, tant qu'il y a des externalités négatives, quel que soit leur degré, les deux voisins se retrouvent dans une situation pire que la situation dont ils jouiraient si aucun des deux n'infligeait des externalités négatives à l'autre. C'est la situation du dilemme du prisonnier. On peut la schématiser ainsi: 


\section{Voisin 1}

\begin{tabular}{l|l|ll}
\cline { 3 - 4 } \multicolumn{1}{c|}{} & $\begin{array}{l}\text { Prérogatives avec } \\
\text { Vestrictions }\end{array}$ & $\begin{array}{l}\text { Prérogatives sans } \\
\text { restrictions }\end{array}$ \\
\cline { 3 - 4 } & $\begin{array}{l}\text { Prérogatives avec } \\
\text { restrictions }\end{array}$ & $\begin{array}{l}\text { (Très satisfait, très } \\
\text { satisfait) }\end{array}$ & $\begin{array}{l}\text { (Perdant, extrêmement } \\
\text { satisfait) }\end{array}$ \\
$\begin{array}{l}\text { Prérogatives sans } \\
\text { restrictions }\end{array}$ & $\begin{array}{l}\text { (Extrêmement } \\
\text { satisfait, perdant) }\end{array}$ & $\begin{array}{l}\text { (Moyennement } \\
\text { satisfait, moyenne- } \\
\text { ment satisfait) }\end{array}$
\end{tabular}

Dans des situations à plusieurs acteurs comme celle-ci, les prérogatives sans restrictions donnent lieu inévitablement à une situation compétitive, et le dilemme du prisonnier surgit. Or il y a un moyen de sortir du dilemme, et c'est en faisant appel à des règles qui sont principalement d'ordre moral. Il serait avantageux pour les deux voisins de se mettre d'accord sur un système de restrictions morales ${ }^{11}$. Ces restrictions seraient d'ordre déontologique, car elles seraient fondamentalement basées sur le respect de l'autre. Dès qu'elles seraient appliquées, ces restrictions limiteraient les externalités nocives et permettraient aux individus d'exercer leurs prérogatives à l'intérieur de ces limites. Tant que chaque voisin peut être confiant que l'autre suivra la règle de leur entente, tous les deux peuvent cohabiter dans le même environnement en étant très satisfait. Ce comportement est requis non seulement pour des raisons morales mais parce que c'est aussi la voie rationnelle ${ }^{12}$.

Ce raisonnement peut être étendu à tout le quartier, où plusieurs personnes interagissent. Nous aurons dans ce cas ce qu'on appelle le dilemme du prisonnier à personnes multiples. La solution est basée sur le même principe: il s'agit de se mettre d'accord sur un ensemble de restrictions qui feront partie d'une stratégie collective de coopération. Ainsi, on pourrait élargir le champ du raisonnement et l'appliquer dans divers domaines d'interaction humaine à l'échelle de la société. Il s'ensuit que dans une société bien ordonnée et juste, chaque liberté accordée à un agent devrait avoir une limite définie non pas par un critère conséquentialiste, mais par un équilibre basé sur la coopération.

L'argument contractualiste nous fournit donc une deuxième raison de définir la portée des prérogatives à la lumière des restrictions déontologiques. Il montre que la justification des prérogatives ne peut pas se faire sans évoquer les restrictions déontologiques. Comme nous l'avons vu aussi dans l'argument précédent, nous aboutissons, cette fois-ci par le dilemme du prisonnier, à la conclusion que les prérogatives ne tiennent pas si elles sont sans restrictions. Ce que montre le dilemme du prisonnier, c'est qu'il n'y a pas moyen de disposer

11. Cf. Gauthier, 1987, chap. 5 et 6.

12. Cf. Heath, 1997. 
des prérogatives s'il n'y a pas de restrictions déontologiques. Et encore, comme dans l'argument précédent, si ces considérations sont fondées, un agent ne peut pas justifier des prérogatives personnelles tout en rejetant les restrictions déontologiques. Cet argument implique que les prérogatives permises dans le cadre d'une théorie morale doivent avoir une portée plus limitée que ce qui est impliqué par la position de Scheffler.

\section{Objection et réponse}

Une objection aux deux arguments que j’ai proposés peut être anticipée. On pourrait dire contre ma thèse que celle-ci réduit considérablement l'espace personnel dans lequel l'agent moral exerce ses prérogatives. Ma thèse garantit un espace personnel aux agents, mais elle demande aussi à l'agent d'abandonner certains de ses projets et ambitions, ou de les modifier, s'ils se trouvent en contradiction avec les restrictions déontologiques. Le contradicteur répliquera que nous avons souvent des projets et engagements sur lesquels nous veillons, et qu'il est parfois très difficile de les abandonner. Un des principes méta-éthiques veut qu'une exigence morale doit être possible à réaliser. S'il est très difficile d'abandonner certains des projets et engagements qui nous tiennent à cœur, cela ne rend-il pas ma thèse vulnérable au reproche qu'elle est trop exigeante?

Pour répondre à cette question, il faut essayer de comprendre ce qu'on entend par «difficile» ou «impossible» au point de vue psychologique, et les contraintes que cela risque de poser quant aux exigences normatives. Si d'aucuns trouvent que certaines restrictions sont fondées sur des principes éthiques trop difficiles, il ne s'ensuit pas que la théorie normative demande quelque chose d'impossible. Cela montre que dans beaucoup de cas les standards moraux des gens sont très bas. Mais cette constatation n'est pas une nouveauté. En fait, la raison pour laquelle il semble important de développer une théorie morale plausible et d'essayer de la mettre en pratique dans les interactions humaines et le fonctionnement de la société, c'est justement que le monde n'est pas toujours naturellement juste. Nous avons un jugement sur la façon dont l'interaction humaine et la structure de la société peuvent s'améliorer à la lumière des principes ou des idéaux. Il est implicite que l'état des choses ne satisfait pas ces idéaux et qu'une amélioration à la lumière des principes requiert un effort. Bien sûr, il y a une limite à ce qu'on peut exiger d'une personne. Mais il ne s'ensuit pas, dans la thèse de dépendance que je défends ici, que la notion de restriction déontologique va à l'encontre de nos capacités psychologiques. En fait, l'instabilité morale et rationnelle dans l'option qui consiste à justifier les prérogatives sans restrictions à l'intérieur des contraintes conséquentialistes semble être beaucoup plus intenable que l'équilibre entre les libertés et les restrictions découlant de la thèse de dépendance. 


\section{Conclusion}

Mon objectif dans ce texte était de démontrer, à l'encontre de Scheffler, que les prérogatives ne peuvent pas se justifier sans que l'on fasse appel aux restrictions déontologiques. Elles dépendent de ces restrictions. J'ai offert deux arguments afin de soutenir cette thèse: l'argument par appel aux droits fondamentaux et l'argument contractualiste. Le premier argument vise à démontrer qu'effectivement, si les intérêts des individus constituent le point de départ de la justification des prérogatives personnelles, ils peuvent fonder des droits. Mais la protection des droits impose des restrictions à autrui. Comme ce sont des droits accordés à tous les individus, il est nécessaire de posséder un système de libertés et de restrictions équilibré. Le deuxième argument focalise sur les actions interactives à plusieurs acteurs. Quand on tient compte de cette dimension, les prérogatives engendrent le dilemme du prisonnier qui nécessite un système de restrictions déontologiques comme base de coopération rationnelle. Les deux arguments convergent vers la conclusion que les prérogatives dépendent des restrictions et qu'un agent ne peut disposer de prérogatives sans devoir accepter en même temps des restrictions déontologiques.

\section{Références}

Das, Ramon. «Prerogatives without Restrictions? ", Philosophical Studies, 99, 2000, p. 347-372.

Feinberg, Joel. "The Rights of Animals and Unborn Generations", Justice and the Bounds of Liberty: Essays in Social Philosophy. Princeton, N. J., Princeton University Press, 1980.

Gauthier, David. Morals by Agreement. Oxford, Oxford University Press, 1987.

Heath, Joseph. "Foundationalism and Practical Reason », Mind, 106, 1997, p. 451474.

Kagan, Shelley. «Does Consequentialism Demand Too Much?», Philosophy and Public Affairs, 13, 1984, p. 239-254.

Scheffler, Samuel. The Rejection of Consequentialism. Oxford, Clarendon Press, 1982.

Scheffler, Samuel. "Prerogatives without Restrictions ", Philosophical Perspectives, vol. 6, Ethics, 1992, p. 377-397.

Scheffler, Samuel. Boundaries and Allegiances: Problems of Justice and Responsibility in Liberal Thought, Oxford, OUP, 2001. 